\title{
Self-Referenced, Trimmed and Compensated RF CMOS Harmonic Oscillators as Monolithic Frequency Generators
}

\author{
Michael S. McCorquodale \\ Mobius Microsystems, Inc. \\ Sunnyvale, CA USA 94086 \\ mccorquodale@mobiusmicro.com
}

\begin{abstract}
Self-referenced, trimmed and temperature-compensated radio frequency (RF) CMOS $L C$, or harmonic, oscillators (CHOs) are presented as high-accuracy and low-jitter monolithic frequency generators. CHOs are discussed within the context of recent efforts toward replacement of piezoelectric frequency references with silicon MEMS technology. In contrast, CHOs are self-referenced solid-state oscillators which can be fabricated in a standard microelectronic process technology. The CHO architecture and recent implementations are presented. Frequency- and time-domain performance of $\mathrm{CHOs}$ is reported and compared to the incumbent piezoelectric oscillators and emerging MEMS-referenced synthesizers. It is shown that $\mathrm{CHOs}$ achieve frequency error as low as $\pm 26 \mathrm{ppm}$ over $90^{\circ} \mathrm{C}$ and $1 / 6^{\text {th }}$ the period jitter of MEMS-referenced synthesizers at the same frequency.
\end{abstract}

\section{INTRODUCTION}

Frequency control utilizing piezoelectric references dates back to 1919 [1]. Since then, quartz crystal oscillators (XOs) have become the standard frequency reference in electronic platforms. However, by the 1980's, crystal-referenced solidstate phase-locked loops (PLLs) emerged as the standard technology for synthesizing multiple frequencies and frequencies higher than could be generated with XOs despite the fact that these PLLs degrade short-term stability.

Most recently, FBAR [2] and MEMS microresonators [3] have emerged as technologies suitable for replacement of SAW and BAW quartz references. The motivation has been to eliminate these latter macroscopic components and replace them with the former devices which utilize a technology compatible with standard batch-processed and lithographic techniques employed in the manufacturing of silicon microelectronics. Though these technologies are gaining commercial traction, it is conspicuous that the limits of frequency generation and control in a standard microelectronic technology appear to remain relatively unexplored. In this work, progress in the development of self-referenced, trimmed and temperature-compensated radio frequency (RF) CMOS $L C$, or harmonic, oscillators (CHOs) as monolithic frequency generators is presented. Further, the frequency- and time-domain performance of these devices is benchmarked against both the incumbent piezoelectric oscillators and emerging MEMS-referenced frequency synthesizers.

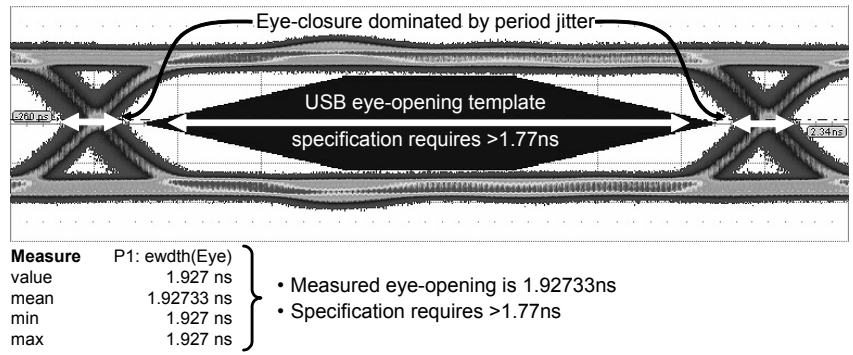

Figure 1. Measured eye-closure in a USB transceiver referenced to a frequency generator with $\pm 100 \mathrm{ppm}$ frequency error. The data show that the measured eye-closure of $156 \mathrm{ps}$ is dominated by period jitter.

\section{TECHNiCAL CONCEPTS}

\section{A. Eye-closure in serial-wire interfaces and total timing error}

Figure 1 illustrates a typical eye diagram measurement for the common serial-wire interface, USB, where the required eye-opening template is shown. Despite the fact that the frequency reference for this transceiver exhibits less than $\pm 100 \mathrm{ppm}$ frequency error (or $0.2 \mathrm{ps}$ for the $480 \mathrm{MHz}$ channelrate clock), the eye-closure is $156 \mathrm{ps}$ which is due to period jitter. As shown, $99.87 \%$ of the eye-closure can be attributed to jitter. Further, it is from this measured eye-closure that the bit error rate is extrapolated. For these reasons, frequency reference jitter is of substantial concern in serial-wire interfaces while frequency error is comparatively less significant.

Considering this phenomenon, the concept of a total timing error can be introduced where both the reference frequency error and the period jitter are considered by superposition or specifically,

$$
\max \left(\delta T / T_{o}\right)=\left(T_{o} \times \max \left(\delta f / f_{o}\right)+\alpha \sigma_{p}\right) / T_{o} .
$$

Here $T_{o}$ is the ideal period, $\delta f l f_{o}$ is the fractional frequency error, $\sigma_{p}$ is the RMS period jitter and $\alpha$ is a scale factor accounting the number of cycles over the observation period as described in [4]. The total timing error will serve as a useful metric for comparing the expected performance of various frequency generators in serial-wire applications. Considering the significant contribution of period jitter to this metric, the relationship of the single sideband (SSB) phase noise power spectral density (PSD) to period jitter is considered next. 


\section{B. Manifestation of phase noise into period jitter}

Consider a unit-amplitude sinusoidal signal with phase noise, $v_{n}(t)=\sin \left(\omega_{o} t+\phi(t)\right)$, where $\omega_{o}$ is the ideal radian frequency and $\phi(t)$ is the phase of $v_{n}$ including phase noise. Next consider two subsequent positive-slope zero-crossings of $v_{n}(t)$ at the time instants $t_{1}$ and $t_{2}$ such that $v_{n}\left(t_{1}\right)=v_{n}\left(t_{2}\right)=0$. At these times, $\omega_{o} t+\phi(t)$ must evaluate to 0 , the phase difference between the two times must be $2 \pi$ and the difference between the two times must be $T_{o}+\delta T$ where $T_{o}=2 \pi / \omega_{o}$ and $\delta T$ is an offset in time from the ideal period due to timing jitter induced by the phase noise. Thus, $\omega_{o}\left(T_{o}+\delta T\right)+\phi\left(t_{2}\right)-\phi\left(t_{1}\right)=2 \pi$. After rearranging and substituting $\omega_{o} T_{o}=2 \pi$, the following is obtained:

$$
\delta T=\frac{1}{\omega_{o}}\left(\phi\left(t_{1}\right)-\phi\left(t_{2}\right)\right)
$$

Now the RMS period jitter can be determined by computing the expected value of the square of the expression in (2).

$$
\delta T_{r m s}^{2}=\frac{1}{\omega_{o}^{2}}\left(E\left[\phi^{2}\left(t_{1}\right)\right]-2 E\left[\phi\left(t_{1}\right) \phi\left(t_{2}\right)\right]+E\left[\phi^{2}\left(t_{2}\right)\right]\right)
$$

Assume that $\phi(t)$ is a stationary stochastic process, thus its autocorrelation is a function of the time difference, $\tau=t_{2}-t_{1}$, or $E\left[\phi\left(t_{1}\right) \phi\left(t_{2}\right)\right]=R_{\phi}\left(t_{2}-t_{1}\right)=R_{\phi}(\tau)$ and (3) becomes:

$$
\delta T_{r m s}^{2}=\frac{1}{\omega_{o}^{2}}\left(2 R_{\phi}(0)-2 R_{\phi}(\tau)\right)
$$

The autocorrelation function and the PSD of $\phi(t)$ are Fourier transform pairs and specifically,

$$
R_{\phi}(\tau)=\int_{0}^{\infty} S_{\phi}\left(f_{m}\right) \cos 2 \pi f_{m} \tau d f_{m}
$$

Using (5) and substituting into (4) yields,

$$
\delta T_{r m s}^{2}=\frac{2}{\omega_{o}^{2}} \int_{0}^{\infty} S_{\phi}\left(f_{m}\right)\left(2 \sin ^{2} \pi f_{m} \tau\right) d f_{m},
$$

where the identity, $1-\cos 2 u=2 \sin ^{2} u$, has been employed. Lastly substitute $S_{\phi}\left(f_{m}\right) \equiv 2\left(N_{o} / P_{o}\right)_{f_{m}}$, as defined in [5], where $\left(N_{o} / P_{o}\right)_{f_{m}}$ is the SSB phase noise PSD. (6) becomes,

$$
\delta T_{r m s}=\sigma_{p}=\sqrt{\frac{8}{\omega_{o}^{2}} \int_{0}^{\infty}\left(\frac{N_{o}}{P_{o}}\right)_{f_{m}} \sin ^{2} \pi f_{m} \tau d f_{m}}
$$

where the notation $\sigma_{p}$ has been reintroduced for the RMS period jitter. Expression (7) is of the same form of the expressions in [6] and [7]. The practical upper limit of the integral is bounded by a downstream PLL, the bandwidth (BW) of the system or the $\mathrm{BW}$ of the measurement instrumentation.

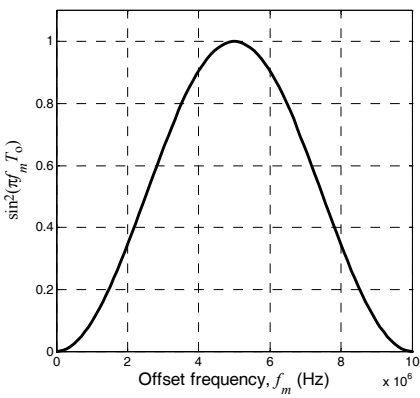

(a)

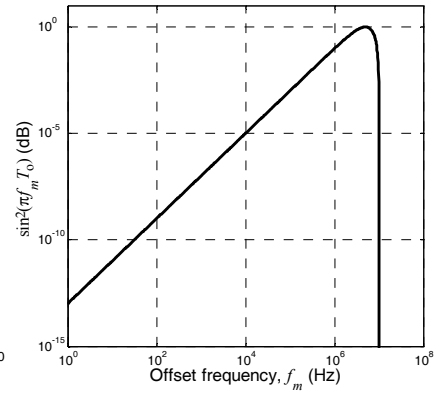

(b)
Figure 2. $\sin ^{2}\left(\pi f_{m} T_{o}\right)$ integration mask against offset frequency (a) on a linearlinear scale (b) on a log-log scale

Consider the $\sin ^{2}\left(\pi f_{m} T_{o}\right)$ masking function in (7) and as shown in Figure 2(a) for a $10 \mathrm{MHz}$ reference oscillator. Here the peak is at an offset equal to half of the oscillation frequency while nulls are at $f_{m}=0$ and $f_{m}=f_{o}$. Next consider that phase noise is typically measured on a log scale. Figure 2(b) illustrates how significantly the close-to-carrier (CTC) phase noise is attenuated by the trigonometric function in (7). At $10 \mathrm{kHz}$ offset, for example, the phase noise is attenuated by $50 \mathrm{~dB}$. Thus, (7) shows that when phase noise is converted to period jitter the CTC phase noise is significantly attenuated and the far-from-carrier (FFC) phase noise is pronounced.

\section{Practical timing implementations}

Practical implementations of frequency generators often include PLL synthesizers which serve to multiply the reference frequency. It is well-known that this linear multiplication by $N$ increases the phase noise power quadratically such that within the PLL loop BW, the phase noise of the reference oscillator is increased by $10 \log _{10}\left(N^{2}\right)$. Outside the PLL loop $\mathrm{BW}$, the phase noise output path tracks the voltage-controlled oscillator (VCO) which exhibits substantially higher phase noise than the high quality $(Q)$ factor reference, be it a crystal or MEMS reference. Further, from (7) it is clear that this noise will contribute more substantially to the jitter and the total timing error. These concepts are illustrated in Figure 3.

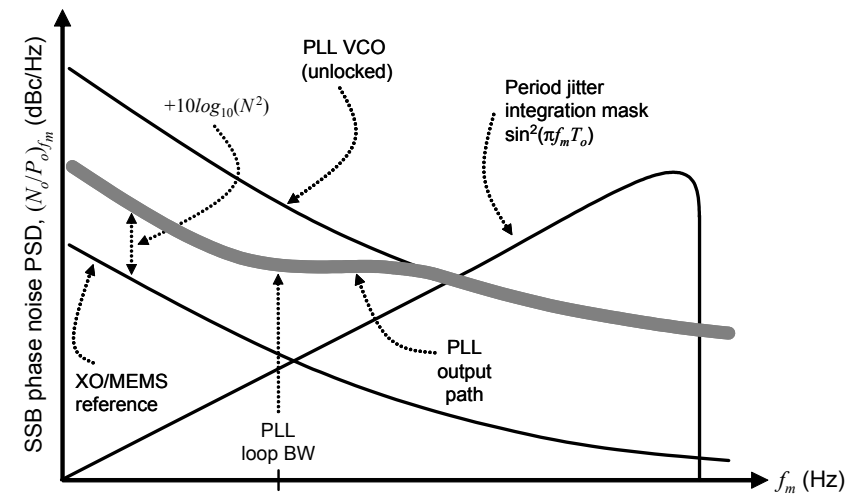

Figure 3. Visualizing the typical PLL synthesizer where the CTC phase noise is shifted by $10 \log _{10}\left(N^{2}\right)$ due to frequency multiplication by $N$ and the FFC phase noise tracks the output VCO. 


\section{CHO ARCHITECTURE AND IMPLEMENTATIONS}

\section{A. CHO Architecture}

Having recognized the aforementioned deleterious effects in frequency synthesis, the $\mathrm{CHO}$ architecture was conceived to achieve low period jitter, low total timing error and high frequency accuracy. Further, an objective was to develop a monolithic, self-referenced, solid-state frequency generator. These objectives are achieved with an RF $L C$ oscillator (LCO). Using a reference oscillator at RF enables frequency division by $N$ to the application frequency which reduces phase noise by $10 \log _{10}\left(N^{2}\right)$ rather than accumulating it as in the PLL. Further, the free-running LCO will exhibit much lower FFC phase noise than a PLL synthesizer. Correspondingly, it is expected to exhibit much lower period jitter. Lastly, precision analog techniques enable frequency trimming and temperature compensation to ensure frequency accuracy.

A typical $\mathrm{CHO}$ architecture is shown in Figure 4. The LCO oscillates at $960 \mathrm{MHz}$ where the tank $Q$ is approximately 10. A binary-weighted 13-bit thin-film switched-capacitor array enables the oscillation frequency to be trimmed at test. A temperature-dependent voltage, $v_{c t r l}(T)$, drives the backgate of a programmable array of varactors and achieves temperaturecompensation of the LCO which is dominated by the coil loss as described in [4]. Disabled varactors are switched to the power supply. Amplitude and common-mode detectors modulate the current into and out of the LCO core respectively. These control loops mitigate frequency drift due to hot carrier and oxide breakdown effects.

The chip-level implementation of the $\mathrm{CHO}$ in [8] is shown in Figure 6. A bandgap-referenced linear regulator biases the $\mathrm{CHO}$, differential-to-single-ended (D2S) converter and the frequency dividers. The compensating signal, $v_{c t r l}(T)$, is generated from diode-referenced currents which are complementary and proportional to absolute temperature, $I_{C T A T}$ and $I_{P T A T}$ respectively. The temperature-dependent slope of $v_{c t r l}(T)$ is pro-

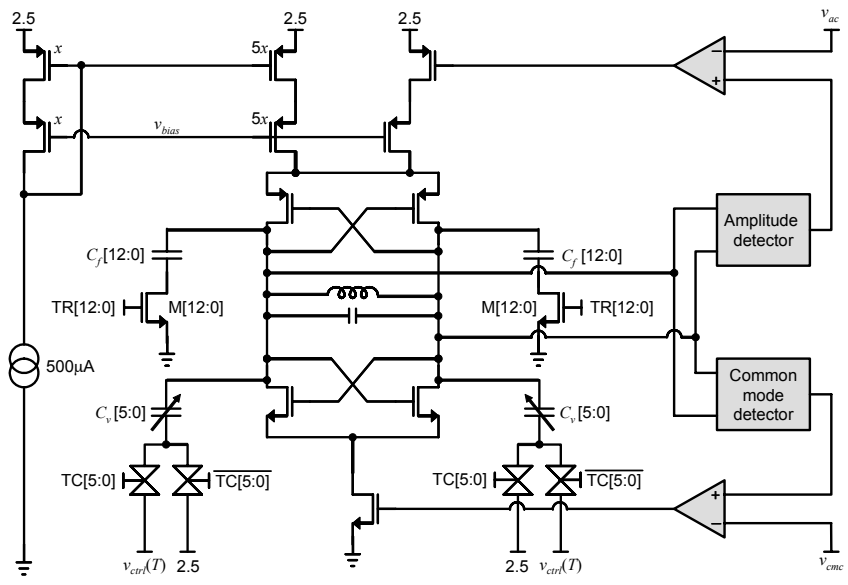

Figure 4. Schematic of the $\mathrm{CHO}$ illustrating the 13-bit fixed capacitor array for frequency trimming, $C_{f}[12: 0]$, the varactor temperature compensation array, $C_{v}[5: 0]$ and amplitude and common mode control loops [8].

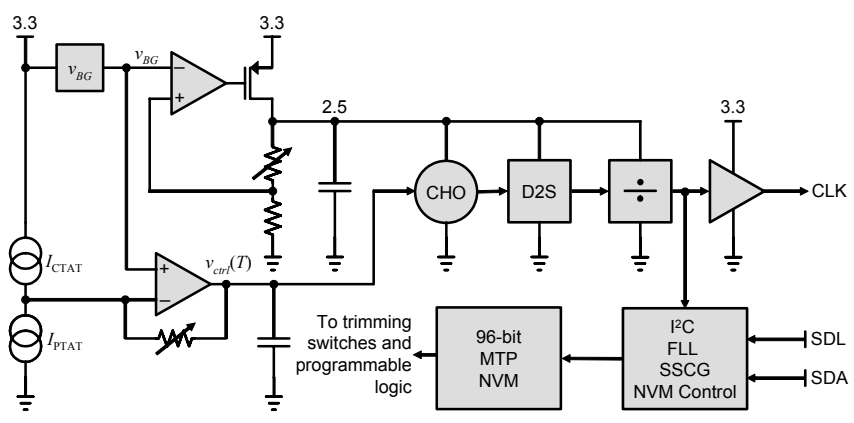

Figure 6. Chip-level architecture of the CHO implementation in [8].

grammed via the variable resistor in the transimpedance amplifier. Lastly, an $\mathrm{I}^{2} \mathrm{C}$ interface serves to program the device while all trimming and configuration coefficients are stored in non-volatile memory (NVM).

\section{B. Recently published CHO implementations}

Original commercial implementations of the $\mathrm{CHO}$ appeared as intellectual property (IP) macros for USB as described in [4]. The macro micrograph is shown in Figure 7. The first component implementation of the $\mathrm{CHO}$ was reported in [8] and the die micrograph is shown in Figure 8. This later component implementation was developed for a broader range of serial-wire applications including S-ATA and PCI. Further, the device in [8] supports spread spectrum clock generation (SSCG) to reduce electromagnetic interference.

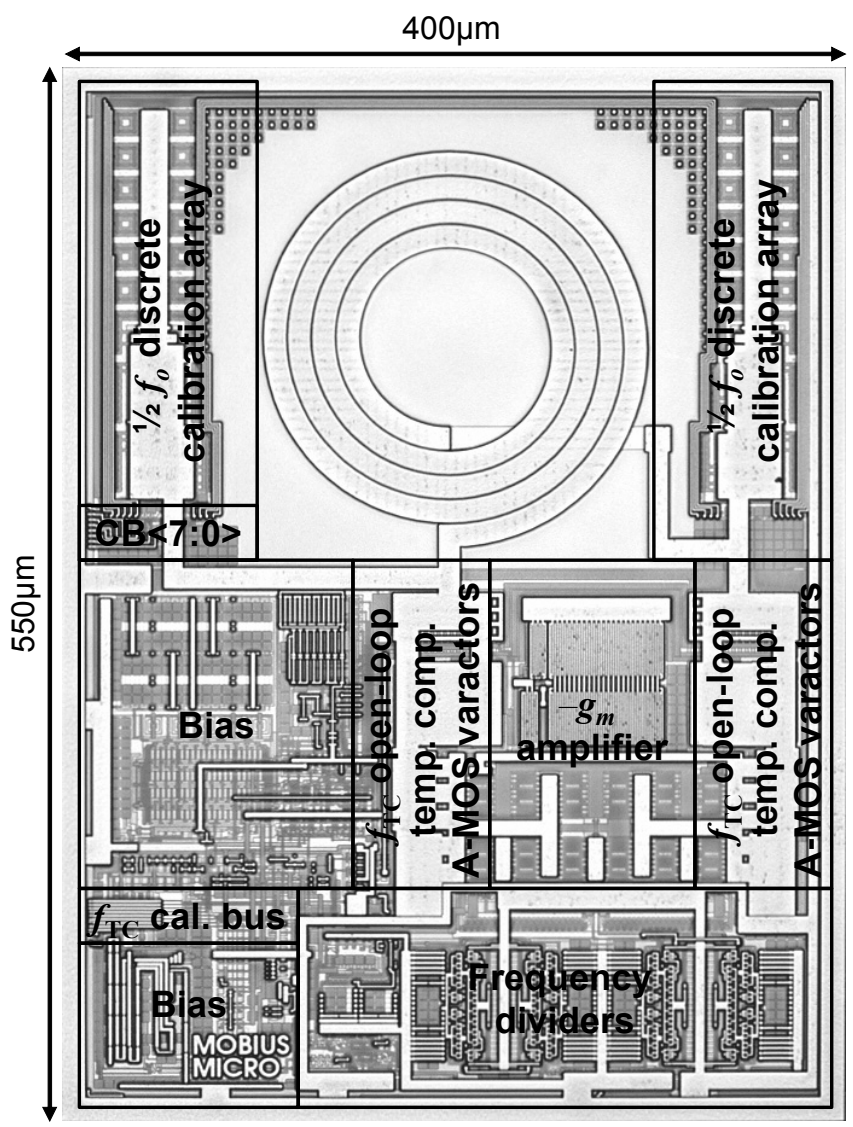

Figure 7. IP macro micrograph of the $12 \mathrm{MHz}$ CHO for USB [4]. 


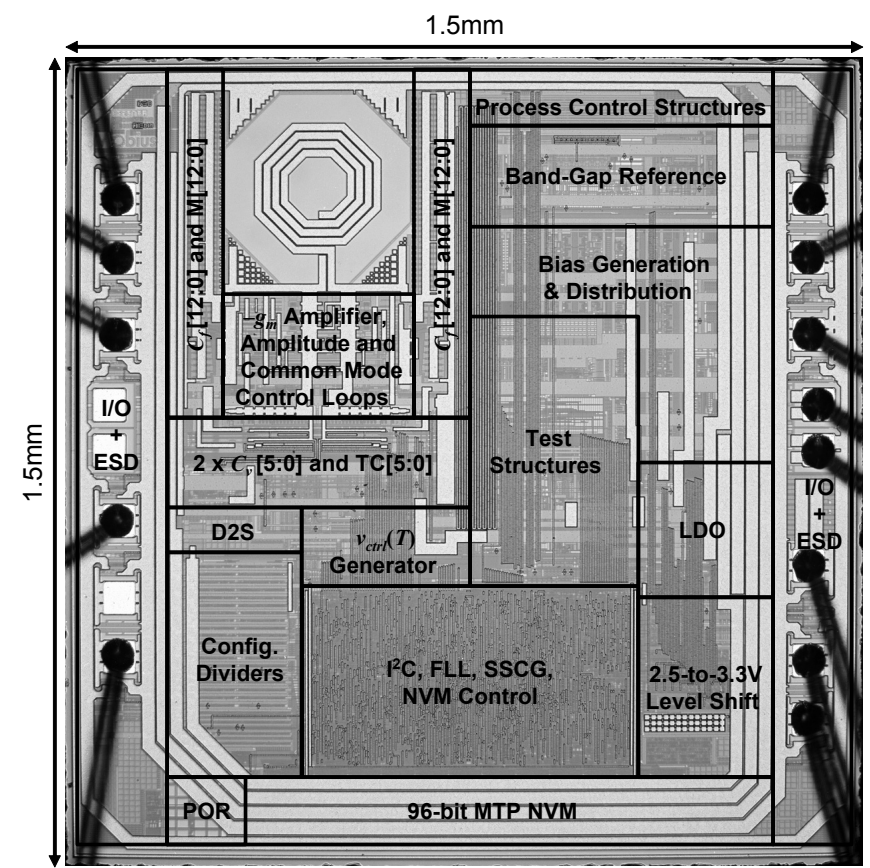

Figure 8. Die micrograph of the general-purpose CHO including SSCG [8].

\section{Measured Performance}

The CHOs in [4] and [8] were tested with 6 different commercially-available frequency generators including the following: $24 \mathrm{MHz} \mathrm{XO}, 24 \mathrm{MHz} \mathrm{XO}-\mathrm{PLL}, 12 \mathrm{MHz}$ ceramic oscillator, 20MHz MEMS-PLL and 12MHz MEMS-PLL. The MEMS-referenced devices were sourced from two different vendors. Tests include total frequency inaccuracy, SSB phase noise PSD, period and cycle-to-cycle timing jitter and total timing error.

\section{A. Total frequency inaccuracy}

All devices were mounted to FR4 printed circuit boards for environmental testing and placed in a temperature chamber where the frequency was measured from $-10^{\circ} \mathrm{C}$ to $80^{\circ} \mathrm{C}$ in $10^{\circ}$ increments. Temperature accuracy of the chamber is within $2^{\circ} \mathrm{C}$. Soak times were 10 minutes per captured data point. Additionally, for the $\mathrm{CHO}$ in [8], the power supply was modulated by $\pm 10 \%$ from nominal at each temperature point. The $\mathrm{CHO}$ in [4] and the XO-PLL were not included. Frequency inaccuracy was measured with a frequency counter and a 1s gate time. Results are shown in Figure 9.

Results show that the ceramic oscillator exhibits a comparatively high temperature coefficient of approximately $\pm 3200 \mathrm{ppm}$. The XO exhibited less than $\pm 10 \mathrm{ppm}$ error while the MEMS-PLLs both achieve below $\pm 50 \mathrm{ppm}$ error. The CHO maintains $\pm 26 \mathrm{ppm}$ error over temperature and $\pm 10 \%$ variation in the power supply. This compensated performance is the best achieved in silicon to date. Typical production performance is $\pm 200 \mathrm{ppm}$ which is limited by test time and associated cost. However, it is feasible to trim the compensation circuity for any device to the accuracy shown in Figure 9.

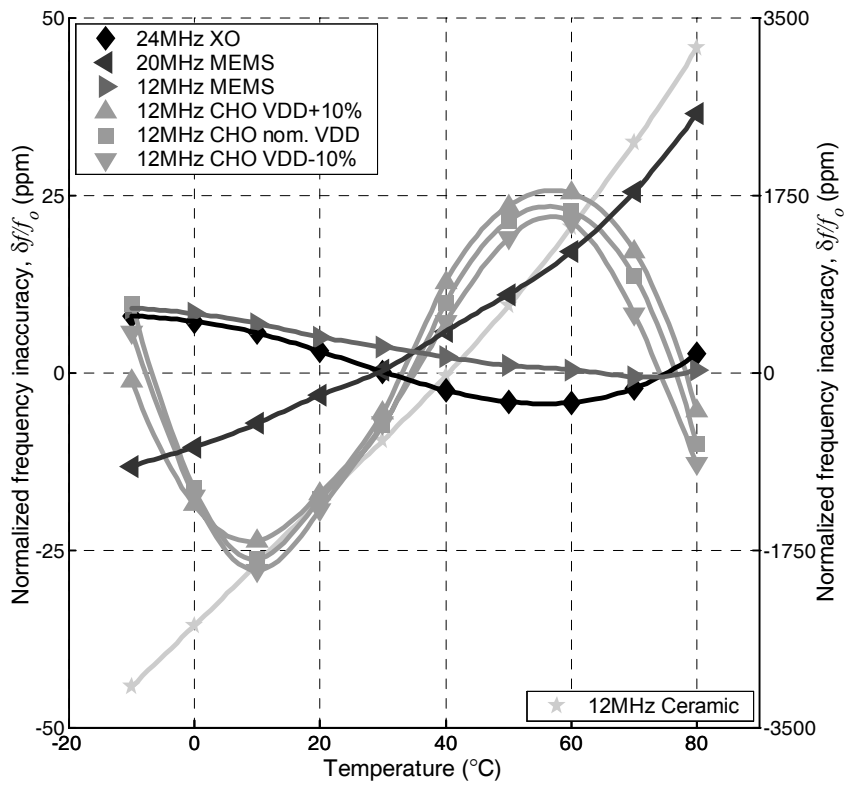

Figure 9. Measured frequency inaccuracy normalized to the ideal frequency against temperature for all tested frequency generators.

\section{B. SSB phase noise PSD}

The SSB phase noise PSD was measured using a signal source analyzer with a low noise floor. Results are shown in Figure 10. Here the XO and XO-PLL exhibit the lowest CTC phase noise. The ceramic oscillator is higher because its $Q$ factor is lower than the $\mathrm{XO}$. Though high- $Q$, the MEMS-referenced devices exhibit relatively high CTC phase noise, due to frequency multiplication, and high FFC phase noise as the

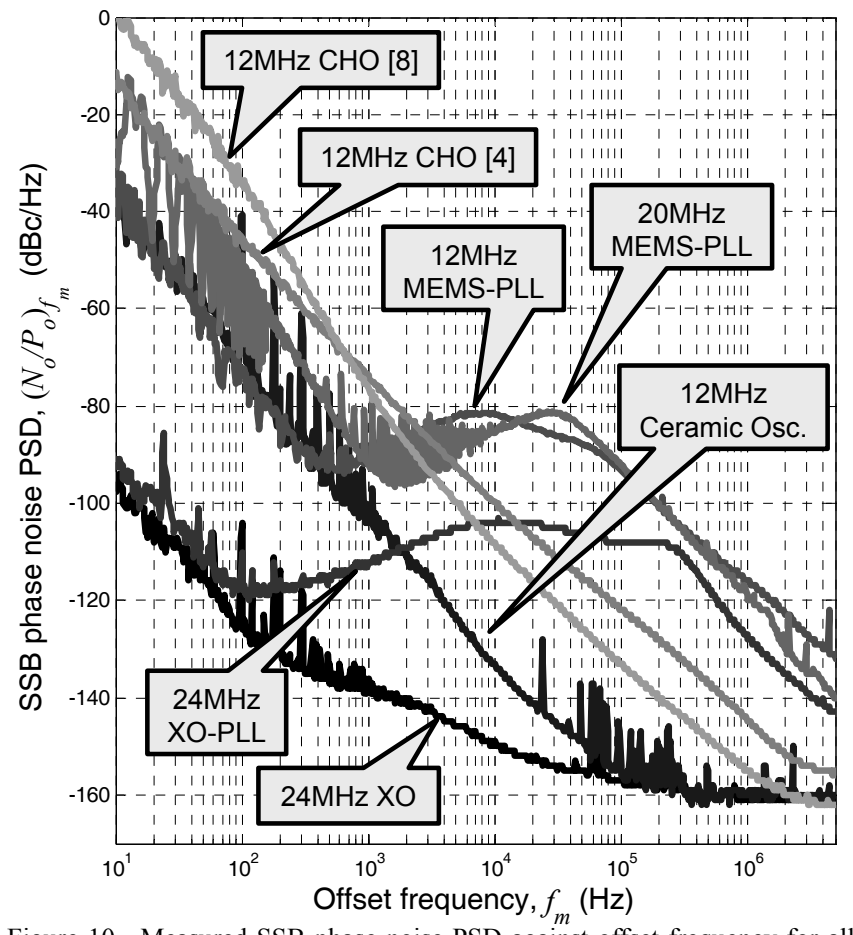

Figure 10. Measured SSB phase noise PSD against offset frequency for all tested frequency generators. 


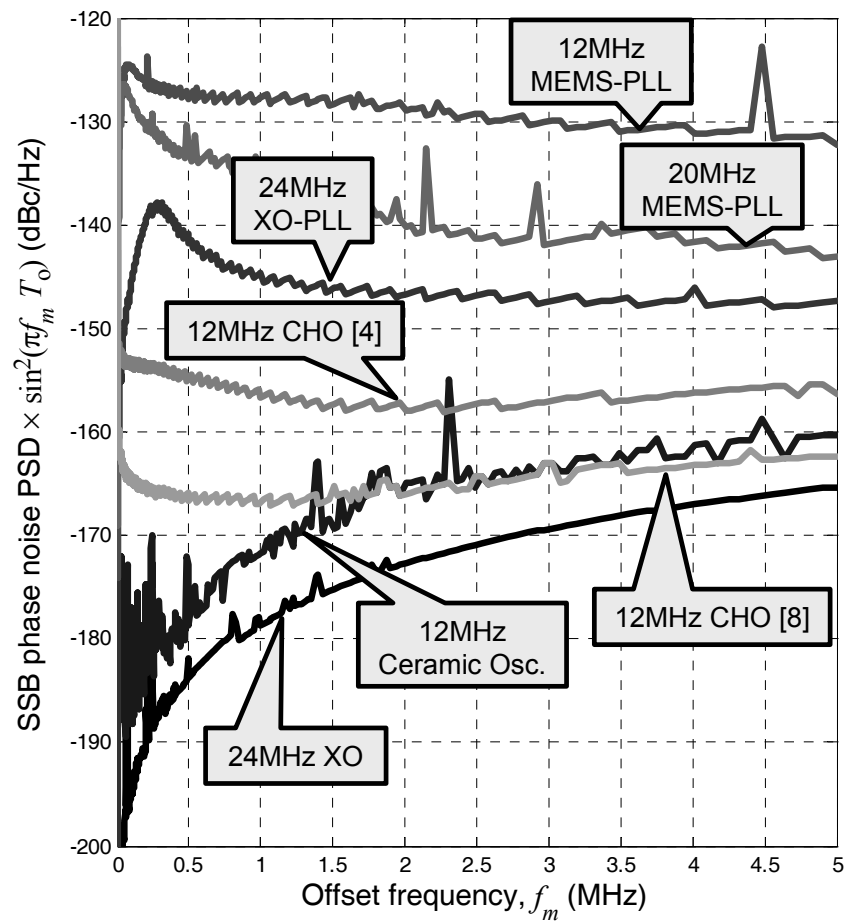

Figure 11. Measured SSB phase noise PSD projected onto $\sin ^{2}\left(\pi f_{m} T_{o}\right)$.

output VCO is a ring oscillator in both implementations. In contrast, the $\mathrm{CHO}$ implementations exhibit CTC phase noise that is comparable to the MEMS-based implementations while the CHOs exhibit substantially lower phase noise FFC.

The phase noise data measured in Figure 10 were exported and projected onto the $\sin ^{2}\left(\pi f_{m} T_{o}\right)$ mask in (7) and are shown in Figure 11 in $\mathrm{dBc} / \mathrm{Hz}$ on a linear frequency scale. As expected, the CTC phase noise is attenuated and the FFC phase noise levels are pronounced. Both $\mathrm{CHO}$ implementations exhibit substantially lower noise than any of the PLL synthesizers when projected onto the trigonometric integration mask. Further, the FFC phase noise for the $\mathrm{CHO}$ in [8] approaches the XO FFC despite the fact that the XO operates at twice the frequency of the CHO. From these results, it is expected that the period jitter and total timing error for the $\mathrm{CHO}$ should be comparable to, or better than, the $\mathrm{XO}$ while the PLL synthesizers will exhibit the highest jitter.

\section{Period and cycle-to-cycle timing jitter}

Period and cycle-to-cycle jitter were measured using a $10 \mathrm{GSa} / \mathrm{s}$ real-time digital sampling oscilloscope (DSO). Results are shown in Figure 12. Amplitude and reference levels were set for each device such that the input signal spanned
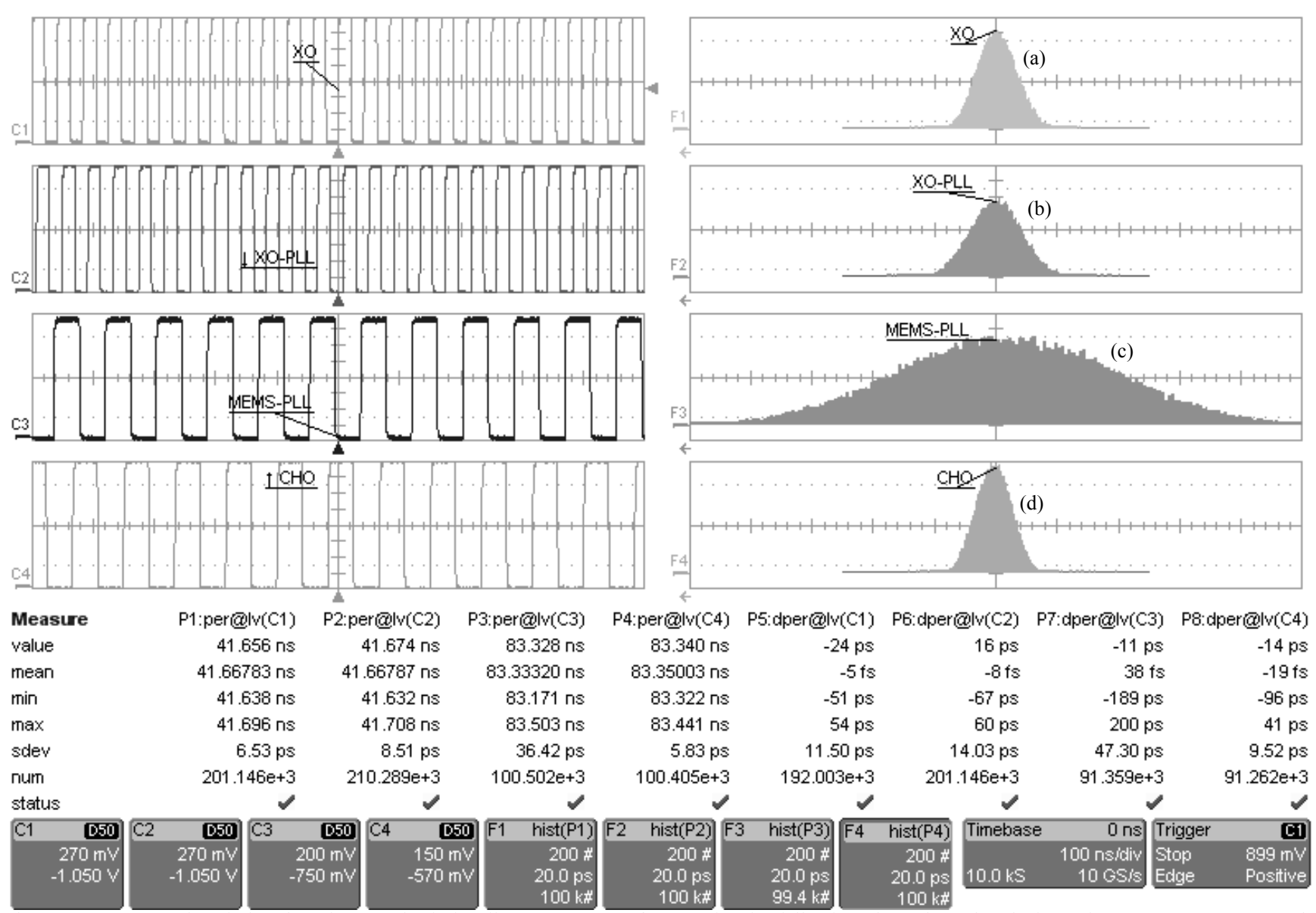

Figure 12. Measured period and cycle-to-cycle timing jitter on a 10 GSa/s DSO for the following channel-number:device pairs: (a) $\mathrm{C} 1: 24 \mathrm{MHz} \mathrm{XO}$ (b) C2:24MHz XO-PLL (c) C3:12MHz MEMS-PLL and (d) C4:12MHz CHO [8]. Period jitter is reported in the columns labeled "per@lv." Cycle-to-cycle jitter is reported in the columns labeled "dper@lv." RMS values are contained in the row labeled "sdev." All histograms are shown on the same scale. 
Table I. Summary of Computed Total Timing ERror Based on Total Frequency InaCCuracy and RMS Period JitTer

\begin{tabular}{|c|c|c|c|c|c|c|c|}
\hline Technology & $\begin{array}{c}f_{o} \\
(\mathbf{M H z})\end{array}$ & $\begin{array}{c}\max \left(\delta f \mid f_{o}\right) \\
(p p m)\end{array}$ & $\begin{array}{c}T_{o} \times \max \left(\delta f \mid f_{o}\right) \\
(\mathrm{ps})\end{array}$ & $\begin{array}{c}\sigma_{p} \\
\left(\mathrm{ps}_{\mathrm{rms}}\right)\end{array}$ & $\begin{array}{c}\alpha \sigma_{p}, \alpha=14.1 \\
\left(\mathbf{p s}_{\mathrm{pp}}\right)\end{array}$ & $\begin{array}{c}\max \left(\delta T / T_{o}\right) \\
\quad(p p m)\end{array}$ & $\underset{(\%)}{\alpha \sigma_{p} / \max \left(\delta T / T_{o}\right)}$ \\
\hline $\mathrm{XO}$ & 24 & 8 & 0.33 & 6.53 & 92.07 & 2218 & 99.6 \\
\hline XO-PLL & 24 & 8 & 0.33 & 8.51 & 120.00 & 2888 & 99.7 \\
\hline Ceramic Osc. & 12 & 3202 & 267 & 6.52 & 91.93 & 4305 & 25.6 \\
\hline MEMS-PLL & 20 & 37 & 1.85 & 12.16 & 171.46 & 3466 & 98.9 \\
\hline MEMS-PLL & 12 & 9 & 0.75 & 36.42 & 513.52 & 6171 & 99.9 \\
\hline $\mathrm{CHO}[4]$ & 12 & 400 & 33.3 & 6.41 & 90.38 & 1485 & 73.1 \\
\hline $\mathrm{CHO}[8]$ & 12 & 26 & 2.17 & 5.83 & 82.20 & 1012 & 97.4 \\
\hline
\end{tabular}

the entire dynamic range of the front-end data converter on the DSO. The measurements were band-limited only by the BW of the instrument. As shown, $100 \mathrm{kSa}$ of the period were captured for each device. Results are shown for: $24 \mathrm{MHz} \mathrm{XO}$, 24MHz XO-PLL, 12MHz MEMS-PLL and 12MHz CHO [8].

The data show that the PLL degrades the RMS period jitter of the XO from $6.53 \mathrm{ps}_{\mathrm{rms}}$ to $8.51 \mathrm{ps}_{\mathrm{rms}}$. The MEMS-PLL period jitter is $36.42 \mathrm{ps}_{\mathrm{rms}}$ while the $\mathrm{CHO}$, at the same frequency, exhibits less than $1 / 6^{\text {th }}$ of the period jitter at $5.83 \mathrm{ps}_{\mathrm{rms}}$ which is achieved due to the low FFC phase noise. Despite the fact that the silicon resonators utilized in the MEMS-PLL device exhibit $Q$-factors on the order of 10,000 or higher, the timing jitter of the synthesized signal is comparatively high due to both frequency multiplication within the loop BW of the PLL and high FFC phase noise outside the loop BW of the PLL where the output phase noise path tracks the VCO.

These results are summarized in Table I where the total timing error, from (1), is included. The scaling factor, $\alpha$, originates from the fact that the period jitter is an unbounded random variable with a Normal distribution. Thus, it is common to specify the peak-to-peak (pp) jitter by scaling the RMS jitter based upon an observation interval as described in [4]. In Table I, the interval is $10^{12}$ cycles, which is a common specification for serial-wire interfaces and which corresponds to the scale factor $\alpha=14.1$. Here it can be seen that both $\mathrm{CHO}$ implementations exhibit the lowest fractional total timing error despite the fact that for [4], the nominal frequency error can be as high as $400 \mathrm{ppm}$.

\section{CONCLUSION}

CHOs were presented as high-accuracy and low-jitter selfreferenced monolithic frequency generators which are implemented in a standard microelectronic process technology. Motivating technical concepts included consideration of eyeclosure in serial-wire interfaces which was shown to be dominated by period timing jitter. The $\mathrm{CHO}$ circuit architecture was presented within the context of achieving low period jitter by exploiting the effects of frequency division and by recog- nizing the significant contribution of FFC phase noise to period jitter. Frequency- and time-domain performance of CHOs was reported and compared to the performance of the incumbent piezoelectric oscillators and emerging MEMS-referenced frequency synthesizers. Results showed that despite the fact that the $\mathrm{CHO}$ is referenced to a comparatively low- $Q$ $L C$ resonator, the achieved period jitter is comparable to XOs and over 6 times lower than the MEMS-referenced frequency synthesizer at the same frequency, $12 \mathrm{MHz}$. Further, the $\mathrm{CHO}$ achieves a frequency inaccuracy as low as $\pm 26 \mathrm{ppm}$ over $90^{\circ} \mathrm{C}$ and $\pm 10 \%$ variation in the power supply from nominal.

\section{ACKNOWLEDGEMENT}

The author acknowledges the members of technical staff at Mobius Microsystems who played instrumental roles in the development of the $\mathrm{CHO}$ implementations reported herein.

\section{REFERENCES}

[1] V. E. Bottom, "A History of the quartz crystal industry in the USA," in Proc. Annual Frequency Control Symposium, Philadelphia, Pennsylvania, 1981, pp. 3-12.

[2] R. C. Ruby, P. Bradley, Y. Oshmyansky, and A. Chien, "Thin film bulk wave acoustic resonators (FBAR) for wireless applications," in Proc. IEEE Int. Ultrasonics Symposium, Atlanta, GA, 2001, pp. 813-821.

[3] C. T.-C. Nguyen, "MEMS technology for timing and frequency control," IEEE Trans. on Ultrasonics, Ferroelectrics, and Frequency Control, vol. 54, no. 2, Feb. 2007, pp. 251-270.

[4] M. S. McCorquodale, et al., "A Monolithic and self-referenced RF LC clock generator compliant with USB 2.0," IEEE J. of Solid State Circuits, vol. 42, no. 2, Feb. 2007, pp. 385-399.

[5] E. S. Ferre-Pikal, et al., "Draft revision of the IEEE STD 1139-1988 standard definitions of physical quantities for fundamental frequency and time metrology-random instabilities," in Proc. IEEE Int. Frequency Control Symposium, Orlando, FL, 1997, pp. 338-357.

[6] I. Zamek and S. Zamek, "Crystal oscillators' jitter measurements and its estimation of phase noise," in Proc. IEEE Int. Frequency Control Symposium and PDA Exhibition, 2003, pp. 547-555.

[7] A. Hajimiri, S. Limotyrakis, and T. H. Lee, "Jitter and phase noise in ring oscillators," IEEE J. of Solid State Circuits, vol. 34, no. 2, June 1999, pp. 790-804.

[8] M. S. McCorquodale, et al., "A $0.5-480 \mathrm{MHz}$ self-referenced CMOS clock generator with $90 \mathrm{ppm}$ total frequency error and spread spectrum capability" in IEEE Int. Solid-State Circuits Conf. (ISSCC) Dig. Tech. Papers, San Francisco, CA 2008, pp. 350-351, 619. 